\title{
Simuler les dynamiques organisationnelles pour concevoir l'organisation. Comment retisser les liens entre stratégie, organisation et travail?
}

Simulate organizational dynamics to design the organization. How to rewrite the links between strategy, organization and work?

\section{Cédric Dalmasso et Jean-Claude Sardas}

\section{OpenEdition}

\section{Journals}

Édition électronique

URL : http://journals.openedition.org/activites/3147

DOI : 10.4000/activites.3147

ISSN : $1765-2723$

Éditeur

ARPACT - Association Recherches et Pratiques sur les ACTivités

Référence électronique

Cédric Dalmasso et Jean-Claude Sardas, « Simuler les dynamiques organisationnelles pour concevoir l'organisation. Comment retisser les liens entre stratégie, organisation et travail ? ", Activités [En ligne], 15-1 | 2018, mis en ligne le 15 avril 2018, consulté le 02 mai 2019. URL : http:// journals.openedition.org/activites/3147; DOI : 10.4000/activites.3147

Ce document a été généré automatiquement le 2 mai 2019.

\section{(ब) $\odot \Theta \Theta$}

Activités est mis à disposition selon les termes de la licence Creative Commons Attribution - Pas d'Utilisation Commerciale - Pas de Modification 4.0 International. 


\section{Simuler les dynamiques} organisationnelles pour concevoir l'organisation. Comment retisser les liens entre stratégie, organisation et travail?

Simulate organizational dynamics to design the organization. How to rewrite the links between strategy, organization and work?

Cédric Dalmasso et Jean-Claude Sardas

\section{NOTE DE L'ÉDITEUR}

Article soumis le 25/11/2017, accepté le 04/03/2018

\section{Introduction}

1 Cet article se situe dans la perspective des sciences de gestion. Il s'intéresse aux processus dits de design organisationnel et argumente en faveur de démarches de simulation afin d'aider les parties prenantes dans la re-conception de l'organisation et l'analyse de la dynamique organisationnelle. Les formes et l'usage de la simulation en sciences sociales sont variés. Ils dépendent de l'objet que l'on cherche à simuler (une chaine de montage, le travail, le fonctionnement organisationnel), des techniques mobilisées pour simuler (la reproduction physique ou virtuelle de l'objet) et des finalités pour lesquelles on les mobilise (comprendre et/ou agir sur le réel).

2 Lorsque la simulation est utilisée dans un projet visant la transformation d'une activité, avant même de parler de simulation, il est nécessaire d'effectuer un large détour pour 
qu'il n'y ait pas méprise sur la posture et les termes adoptés. Nous procéderons donc à une mise en dialogue entre les conceptions de l'activité en gestion et en ergonomie.

Pour aller d'ores et déjà à l'essentiel, nous partageons, avec la perspective ergonomique, l'idée que l'activité forme un complexe par nature non décomposable aux déterminants multiples. Aussi toute volonté de transformation de celui-ci pose la question des chemins praticables et des démarches à mettre en œuvre pour les arpenter. De notre point de vue, le sentier de transformation de l'activité peut se perdre et s'abimer sur deux écueils : l'empirisme objectiviste qui nous conduirait à croire que les dispositifs formels d'organisation rendent parfaitement compte de l'activité et l'empirisme socio-subjectif qui nous ferait abandonner toute volonté téléologique de changement en nous confinant dans la posture de l'analyste de l'activité. C'est tout l'enjeu de la recherche et de l'intervention en gestion que de s'atteler aux problèmes d'organisation propres aux entreprises et c'est la spécificité de notre posture que de le faire en cherchant, cependant, à y insérer les préoccupations d'activité de travail.

Les démarches permettant d'accompagner la transformation d'une activité productive posent quant à elle le même type de question à un autre niveau. Si toute organisation est un processus plutôt qu'un état ou une structure, est-il alors possible d'établir une distinction, dans la démarche d'accompagnement, entre la conception de l'organisation, l'analyse de son fonctionnement et l'expérimentation? Et quelle que soit la posture adoptée, quelle est la place accordée à la participation dans ce processus ? Ce n'est qu'une fois ces clarifications apportées qu'il devient possible de questionner la place de la simulation dans ce projet de transformation. Les questions qui surgissent alors apparaissent comme l'écho des interrogations précédentes. Si la simulation organisationnelle n'est pas l'œuvre d'un expert en charge d'appliquer une technique mais l'occasion d'engager les salariés dans un projet de connaissance, comment est-il possible d'organiser cette participation et pour quels effets?

Pour explorer ces questions nous présenterons plus loin le déroulement d'une recherche intervention qui a mobilisé une simulation organisationnelle. La recherche porte sur l'internationalisation des activités d'ingénierie et sur le découplage entre l'intention stratégique et l'activité des travailleurs intellectuels. La démarche d'intervention et la mobilisation d'une simulation serait-il alors, à l'heure où le travail semble invisible aux yeux des stratèges, un moyen de retisser les liens entre stratégie, organisation et travail ?

6 Pour aborder cette question nous commencerons par clarifier ce que nous entendons par design organisationnel en positionnant notre démarche "conception et dynamique des organisations " par rapport à "l'enquête dialogique ». Nous expliciterons ensuite la manière dont nous avons organisé la participation des parties prenantes et le type de simulation que nous avons mobilisé. Nous présenterons enfin la recherche intervention et discuterons de la fertilisation croisée entre la simulation et les modes de participations.

\section{Design organisationnel et conception et dynamique des organisations}

\subsection{Que conçoit-on en design organisationnel?}

7 Discuter des démarches de design organisationnel appelle rapidement, du fait de la polysémie du terme organisation, à clarifier, comme nous l'avons dit, la nature de l'objet 
que l'on cherche à analyser et à concevoir. D'un côté, une vision étroite consiste à considérer qu'on peut s'en tenir aux dimensions formelles de l'organisation en ne pensant qu'en termes de structure, de processus et d'outils. C'est ce que Lorino (2009) appelle l'empirisme objectiviste qui réduit le design organisationnel à sa dimension technique, en postulant que les dispositifs formels rendent intégralement compte des dynamiques psychosociales. À l'autre extrémité, toute démarche de conception sur l'organisation serait impossible et nous serions réduits à uniquement observer et analyser les dynamiques psychosociales. Nous tomberions alors dans ce que nous pouvons appeler l'empirisme socio-subjectiviste sans possibilité d'agir de manière raisonnée sur l'organisation.

Pour éviter ces deux écueils il convient d'assumer toute la richesse et la complexité de l'activité. Si elle est par nature non décomposable, que ses déterminants sont multiples et que ses modes d'organisation sont définis par des entités diverses à des niveaux variés du collectifs (Salembier, \& Pavard, 2004) cela signifie alors que les démarches de design organisationnel doivent tenir compte de cette totalité complexe organisée (Ibid.). Certains auteurs parlent d'enquête dialogique (Lorino, 2009) pour souligner que l'organisation est un processus plutôt qu'un état ou une structure. Pour notre part, afin de caractériser notre approche du design organisationnel, nous utilisons les termes de " conception et dynamique des organisations » (CDO en abrégé) (Sardas, Dalmasso, \& Lefebvre, 2011). L'objet dont on vise une évolution est bien l'activité mais ses caractéristiques totale et complexe nous empêchent (et nous empêcheront toujours) de la saisir dans sa plénitude. Nous devons alors assumer que l'activité est inatteignable. Pour autant, toute volonté de transformation n'est pas à proscrire. Il est possible d'agir sur les éléments formels d'organisation d'une activité (la définition formelle de la structure, la définition des rôles, la manière dont s'enchaînent les tâches,...) et de notre point de vue ce sont les seuls leviers d'action dont on dispose sur l'organisation. Ces actions sur les traits formels de l'organisation peuvent être conçues pour orienter, permettre ou faciliter certaines évolutions attendues de l'activité. Mais il n'y aura jamais de complétude du savoir sur l'activité, que ce soit individuellement ou collectivement, et des surprises arriveront toujours. Nous utilisons la notion de dynamique organisationnelle qui représente le fonctionnement organisationnel réel dans ses dimensions humaine, technique et gestionnaire. Cette dynamique ne se réduit pas, loin s'en faut, aux éléments formels qui s'efforcent de l'orienter. Les collectifs et les individus s'engagent, se rebellent, évoluent, se transforment, résistent, se renforcent ou s'épuisent, et les systèmes techniques et gestionnaires peuvent dysfonctionner, s'emballer se bloquer, et induire des effets pervers. Nous allons présenter cette posture particulière, visant à articuler dans une même démarche une approche de conception des traits formels de l'organisation et une approche d'analyse psychosociale.

\subsection{Activité : quel usage de la notion?}

9 On le sent, un détour s'impose par ce qu'il faut entendre par activité. En sciences de gestion, la notion d'activité est peu explorée et discutée, sauf par quelques auteurs (Peyrolle, \& Lorino, 1999). Leurs travaux font le constat que le terme activité peut, en sciences de gestion, être mobilisé pour aborder tout à la fois des questions très globales, comme celles relevant de l'analyse stratégique, et des questions plus micro, traitant de l'activité humaine opératoire. C'est cette seconde acception, ce travail doté d'un sens et 
inscrit dans une organisation, qui nous intéressera ici. À ce niveau, pour Peyrolle et Lorino, l'activité se présente sous un double visage, fonctionnel d'une part et cognitif d'autre part. Dans l'approche fonctionnelle, l'activité se définit par ce qu'elle produit, et constitue une boite noire qui transforme des inputs en output. Ces auteurs font alors remarquer que " cette approche ne peut être épistémologiquement viable que si l'output est normé, stable et mesurable (sinon, le concept d'activité n'a pas de substance)». L'approche cognitive quant à elle aborde l'activité comme un processus de construction de connaissance qui s'opère par l'engagement même dans l'activité. "L'activité ne se réfere plus à un output clairement spécifié mais à un objectif plus global, une contribution à la résolution d'une catégorie de problèmes dans un périmètre délimité » (Ibid.).

10 Peyrolle et Lorino considèrent, en se référant à Lerch (1998), que l'approche la plus opérante (fonctionnelle ou cognitive) dépendrait de la nature de l'activité. L'approche fonctionnelle serait adaptée aux activités normées et répétitives alors que l'approche cognitive aurait un sens sur les activités de type "expérimentation, exploration et diagnostic " dont un des parangons est l'activité de conception. Cette proposition, détentrice d'une utilité pratique certaine, laisse toutefois supposer qu'il est possible de choisir entre l'une ou l'autre des approches pour décrire l'activité. Pourtant même les activités productives stabilisées reposent sur des dynamiques d'apprentissage qu'il est intéressant d'étudier et dont il faut maintenir les conditions de leur pérennité. De la même manière, dans les activités de conception, l'approche cognitive masque l'important travail d'explicitation et d'organisation des différentes étapes du processus de conception en spécifiant à la fois, pour l'ensemble des acteurs impliqués, les livrables attendus et les données nécessaires. Si cette activité est certes cognitive, elle n'en fait pas moins l'objet d'une rationalisation fonctionnelle. Ainsi, s'il est utile de distinguer entre l'approche fonctionnelle et l'approche cognitive ${ }^{1}$, il ne nous semble pas possible de faire l'économie de l'une ou de l'autre. Et nous allons d'ailleurs voir qu'une des spécificités de nos travaux résident dans l'effort d'articulation de ces deux approches.

11 Pour notre part, nous employons le terme activité pour désigner l'objet à analyser et/ou l'objet dont on souhaite faire évoluer le fonctionnement en agissant sur ses modes formels d'organisation. Dans cette optique, cet objet d'étude et d'action n'est ni une entreprise, ni une institution, mais plutôt, en première approche, un processus productif, matériel ou immatériel, qui mobilise des individus, des technologies, des connaissances et des outils de pilotage, processus qui pourra traverser les frontières des entreprises, et dont il faudra définir les limites. Par exemple, on peut raisonner sur une activité de montage automobile d'un véhicule donné, qui sera formellement organisée en ligne d'assemblage dans l'usine du constructeur avec quelques fournisseurs approvisionnant en flux tendu certains sous-ensembles directement sur la ligne. Et cette délimitation conceptuelle qui englobe l'atelier de montage et les fournisseurs, délimitation toujours à définir, aura du sens si le projet consiste par exemple à étudier la possibilité d'implanter en épis sur la chaîne principale des modules autonomes de préparation de ces sous-ensembles.

Pour autant, l'activité n'est pas pour nous uniquement un processus productif, nous voyons également celle-ci (quelle que soit sa nature plus ou moins exploratoire) comme un processus de construction des connaissances et aussi comme un processus de construction de soi dans lequel les individus s'engagent. Ainsi, lorsque nous parlons d'organisation d'une activité, c'est en fait un raccourci pour parler de l'action de définition de l'organisation formelle du processus qui sous-tend cette activité. Nous adoptons un regard fonctionnel sur l'organisation (au sens de l'approche fonctionnelle) 
mais nous gardons à l'esprit que cela ne nous dit rien sur le fonctionnement organisationnel réel du système sociotechnique complexe qui sous-tend l'activité. Nous parlons alors de dynamique organisationnelle pour désigner la réalité du fonctionnement de ce système sociotechnique et la manière dont les individus endossent, habitent et transforment les rôles qui leurs sont proposés ainsi que la manière dont ils pensent, résistent, façonnent (ou échouent à le faire) les conditions de réalisation de l'activité. Ainsi nous sommes en accord avec les distinctions classiques entre organisation formelle et informelle, régulation de contrôle et régulation autonome (Reynaud, 1997) ou encore organisation et travail d'organisation (De Terssac, 2002). Mais nous nous efforçons d'apporter la singularité du regard gestionnaire à cette distinction. Si en définitive il est scientifiquement impossible d'anticiper le réel du travail (Schwartz, 2007) nous n'abandonnons pas une visée de transformation de l'activité et de développement conjoint de l'individu et de l'entreprise. A notre échelle nous participons alors aux débats qui animent la communauté des ergonomes - du courant francophone centré sur l'activité avec qui nous partageons la visée de transformation - sur l'hétérogénéité des finalités visées, allant du développement de l'individu, à celui de l'entreprise et plus largement de la société (Daniellou, \& Béguin, 2004 ; Hubault, \& Bourgeois, 2004).

\section{Participation des parties prenantes et simulation dans le design organisationnel}

\subsection{Quelle démarche de conception de conception organisationnelle?}

Notre approche (nommée «Conception et Dynamique des Organisations ») consiste en une démarche intentionnelle de (re)conception organisationnelle visant à influencer la dynamique organisationnelle. Nous distinguons différentes étapes complémentaires (l'explicitation des finalités visées, l'analyse du fonctionnement actuel, la conception d'un nouveau schéma organisationnel, l'analyse du fonctionnement projeté et l'expérimentation) afin de mettre en évidence le fait que chacune d'entre elles reposent sur des logiques et des savoirs différents. L'enjeu est bien entendu de les articuler si l'on veut comprendre et accompagner le processus de changement. En effet il serait vain de vouloir analyser une dynamique organisationnelle sans avoir à l'esprit les finalités de cette analyse, finalités qui permettent de se donner des orientations, même temporaires, sur ce qui est a priori important et accessoire (et pour qui), sur ce qui est a priori connu et inconnu (et pour qui). En retour, il serait risqué et absurde d'envisager une re-conception formelle de l'organisation sans en amont analyser son fonctionnement pour justement essayer de déterminer ce qui, en fonction des finalités, est important et accessoire, connu et inconnu (et pour qui). Mais malgré ces interactions parfois très fortes entre conception et analyse, il nous semble tout de même important d'opérer une distinction entre ces étapes afin notamment de bien marquer la différence entre ce qui se conçoit, au sens où l'on peut objectivement agir sur certains leviers, et les éléments dont on ne peut qu'observer la dynamique. La culture, la confiance, la reconnaissance, la santé, à des degrés divers, peuvent s'observer et s'analyser mais, de notre point de vue, ne se conçoivent pas. Pour autant l'organisation formelle influence les dynamiques observées. La re-conception provoquera ou contribuera à l'évolution de la dynamique organisationnelle mais toujours de manière non déterministe. C'est cette indétermination 
relative qui justifie la mise en œuvre et le suivi d'une expérimentation car concevoir intentionnellement une nouvelle organisation nous confronte toujours, à plus ou moins grande échelle, à des surprises qui appellent des adaptations, qui elles-mêmes pourront engendrer quelques évolutions allant dans le sens souhaité et de nouvelles surprises.

En opérant une distinction entre l'analyse, la conception et l'expérimentation nous nous situons dans une lignée intellectuelle qui n'abandonne pas l'ambition de transformer la dynamique organisationnelle au travers d'une démarche intentionnelle et rationnelle. $\mathrm{Si}$ en effet nous considérons qu'une dynamique organisationnelle est par nature interactionniste (Lorino, 2009) c'est à dire si nous considérons qu'il existe des relations complexes d'interdépendance entre activités et que ces relations ne peuvent se réduire à des relations input-output, nous pensons tout de même qu'il est possible de progresser dans la conception de schémas d'organisation (au sens où certains schémas sont plus pertinents, cohérents et efficients que d'autres) et que cette progression passe par la distinction et l'explicitation des variables de conception de l'organisation et de leurs liens aux dynamiques organisationnelles. En ce sens nous nous distinguons de la démarche d'enquête dialogique proposée par Lorino (Ibid.) en adoptant une posture plus intervenante. Mais pour autant, nous ne sommes pas si éloignés quant à l'appréhension $\mathrm{du}$ processus de changement organisationnel que nous considérons à la fois comme interactionnel et transactionnel. Il est interactionnel car pour se réaliser il faut que les différents acteurs impliqués interagissent entre eux de toutes sortes de manières (...), ces interactions sont marquées par le sceau de l'hétérogénéité et participent tout à la fois à la transformation de l'activité et à la transformation des acteurs. Il est transactionnel au sens où les interactions au sein du processus doivent (..) être réglées de manière à ce que la transaction globale se réalise dans des conditions satisfaisantes (Ibid.). Il nous semble alors, si l'on adopte ce vocabulaire, que notre démarche s'efforce par rapport à l'enquête dialogique d'étayer de manière plus importante et active la dimension transactionnelle en laissant toutefois une place importante à la dimension participative.

\subsection{Comment étayer la participation dans une démarche de design organisationnel}

15 Notre démarche «conception et dynamique des organisations » s'appuie sur la mise en place d'un dispositif de pilotage dont l'ambition est à la fois de conduire l'expérimentation et de structurer les interactions entre les parties prenantes concernées. A priori, nous préconisons l'intégration de l'ensemble des parties prenantes concernées par le processus de réorganisation (ce qui signifie que le dispositif de pilotage peut être évolutif dans sa composition en fonction de l'évolution du périmètre jugé comme pertinent) et a minima lorsque le périmètre concerné est trop large d'un représentant par partie prenante. Dans notre démarche, l'effort est porté sur la qualité du diagnostic des dynamiques organisationnelles actuelles, sur la pertinence et la cohérence du nouveau schéma organisationnel formel issu de l'étape de conception et sur la qualité de la projection de la future dynamique organisationnelle, projection qui permet d'anticiper autant que possible les avatars de cette future dynamique et de tenter de les corriger. Ce sont ces qualités de diagnostic et de conception qui permettent de faire du dispositif de pilotage un lieu intentionnel d'expérimentation et d'apprentissage.

Mais nous considérons que s'en remettre aux seules capacités des parties prenantes pour faire évoluer l'organisation dans un sens favorable ne suffit pas. Cela ne signifie pas que 
nous disposons en tant que chercheurs d'un savoir surplombant et supérieur. Nous partageons plus humblement avec les participants des méthodes et des grilles de lecture et d'analyse supposées les aider (et nous aider) à rendre intelligible la dynamique de l'organisation à reconcevoir et les liens avec les variables formelles d'organisation. Nous mobilisons trois supports pour étayer la participation. Le premier concerne la démarche CDO elle-même et la distinction déjà évoquée entre analyse, conception et expérimentation. L'enjeu est ici de bien marquer l'importance de la distinction entre ce qui s'analyse, ce qui se conçoit et ce qui s'expérimente. Le deuxième est une méthode d'analyse du comportement des acteurs de l'organisation nommée «Dynamique Identitaire Globale de l'Acteur " (Sardas, Dalmasso, \& Lefebvre, 2011) qui cherche à comprendre la manière dont le rôle formel proposé à un acteur (individuel ou collectif) est habité par ce dernier. Cette méthode d'analyse cherche à élucider les interactions entre les dynamiques physiologique, cognitive, du pouvoir d'agir et subjective et, partant de là, les risques de blocage de la dynamique identitaire globale de l'acteur. Le troisième est une méthode de conception du dessin formel de l'organisation (Ibid.) qui permet de décrire et de mettre en débat un tel dessin; elle distingue les processus opératoires (l'enchainement des opérations), les processus ressources (création et développement des ressources humaines, techniques et de connaissance) et les éléments structurels (macrostructure et rôles). En plus de ces supports nous pouvons selon les besoins concevoir des démarches ad hoc de simulation organisationnelle pour aider les parties prenantes dans le processus de re-conception de l'organisation et d'analyse de la dynamique organisationnelle. Ces méthodes de simulation pouvant se définir comme la mobilisation d'un support conceptuel ou physique permettant de comprendre le fonctionnement d'un système sociotechnique complexe, de l'orienter ou de l'améliorer en anticipant son fonctionnement.

\subsection{La simulation comme extension des capacités de design organisationnel}

17 La simulation organisationnelle n'est pas une méthode éprouvée (Van Belleghem, 2012) comme peut l'être la simulation du travail. Plus novatrice, elle nécessite un effort d'innovation pour développer des supports de simulation permettant de représenter l'organisation à concevoir et d'y jouer le travail futur (Ibid.). Cette méthodologie encore balbutiante doit préciser quelles dimensions de l'organisation sont à prendre en compte, quels sont les différents scénarii à étudier, quelles sont les dimensions de l'activité à simuler et quels supports permettront de le faire. Mobiliser une simulation organisationnelle ravive les débats épistémologiques qui opposent l'approche « experte » et l'approche " participative ». En tant que telle, une méthode de simulation n'appelle pas forcément à la mise en œuvre d'une démarche participative mais les hypothèses implicites qu'elle contient pose tout de même question lorsque l'on souhaite simuler le travail ou l'organisation. Daniellou (1999) éclaire ce débat en explicitant deux grands types de postures que peut adopter l'ergonome (et plus largement selon nous le chercheur en sciences sociales) ainsi que ce que celles-ci permettent ou ne permettent pas d'atteindre. Selon cet auteur (1999), une première position consiste à considérer l'ergonomie comme une technologie qui applique des connaissances fondamentales sur la physiologie et la cognition humaine pour améliorer les conditions de travail. Implicitement, cette approche d'expert, que l'auteur n'appelle pas de ses vœux, présuppose que l'ergonome sache a priori ce qu'est le travail et qu'il évalue un projet de conception sans avoir à 
s'interroger sur sa propre représentation du travail futur. L'autre position est de considérer que l'ergonomie est une pratique qui cherche à favoriser des débats, des processus de conception et des décisions qui prennent en compte la complexité de ce qui se joue dans les situations de travail. L'ergonome se permet alors d'atteindre la densité de l'activité de travail et de nouvelles voies de développement. C'est cette démarche, nécessairement participative, que propose d'adopter Van Belleghem (2012) dans le cadre des simulations organisationnelles. Selon lui, un des effets principaux en est que les salariés engagés dans la démarche construisent non seulement une connaissance fine du contenu du projet (en participant à l'instruction des choix le concernant), mais aussi et surtout de ce qu'il nécessitera de leur part en termes de mobilisations de savoir-faire (avant même sa mise en place effective).

En pratique, sans que la dimension participative soit absente de notre démarche de simulation organisationnelle (et nous reviendrons spécifiquement sur ce point dans notre discussion), elle n'est pas, dans le cas présenté, participative au sens fort du terme tel que l'utilise Van Belleghem. Nous nous situons plutôt dans ce que Salembier et Pavard (2004) appellent la simulation des mécanismes informels de coopération homme-homme qui est une simulation faiblement prédictive utilisée comme moyen d'administration de la preuve. La simulation, qui s'appuie ici sur un modèle empirique synthétique, a pour finalité de traiter d'un aspect particulier de l'activité collective, Il repose sur une base empirique étoffée (...) qui d'un point de vue opérationnel permet d'orienter les décisions de transformation des situations de travail (Ibid.). C'est dans la constitution de cette base empirique étoffée que notre démarche est participative. On opère ensuite par simplification pour établir une simulation et ses résultats nourrissent la discussion et la négociation avec et entre les parties prenantes. Les limites d'une telle approche tiennent à sa nature déterministe et au fait que l'on est rarement surpris des résultats obtenus (nous reviendrons sur ce point en discussion). Les apports résident alors dans l'intelligibilité et l'effort de rigueur dans la description de ce que l'on cherche à simuler.

Venons-en maintenant à présenter une recherche intervention dans laquelle nous avons fait appel à une simulation organisationnelle.

\section{La recherche-intervention et la fertilisation croisée entre la simulation et les modes de participation}

\subsection{Une étude de cas portant sur la création d'une ingénierie mondialement répartie}

20 Le cas d'étude que nous présentons est tiré d'une recherche intervention de longue durée (plusieurs années) au cours de laquelle nous avons été amenés à utiliser une simulation organisationnelle faiblement prédictive. Pour comprendre pourquoi nous en sommes arrivés là, nous devons revenir aux origines de la transformation engagée par l'entreprise partenaire. Nous aborderons successivement l'intention stratégique fixée par le sommet stratégique de l'entreprise, la stratégie de déploiement (conçue globalement par le sommet stratégique de l'entreprise et dans le détail par le management intermédiaire) et la dynamique organisationnelle telle que vécue par les travailleurs intellectuels.

21 L'intention stratégique était formulée très clairement. Il s'agissait pour l'entreprise d'accroître ses ressources d'ingénierie de 30 \% à l'international afin d'atteindre un double objectif : d'une part, partir à la conquête des marchés internationaux en proposant des 
produits plus adaptés aux spécificités des consommateurs locaux et d'autre part, accroître l'offre globale de nouveaux produits sans que l'accroissement des coûts de conception ne viennent réduire la marge de l'entreprise. Pour tenir ces différents objectifs, la solution envisagée consistait à recruter les nouvelles ressources de conception dans des pays à bas coût en créant de nouveaux centres d'ingénierie. Ces nouvelles entités avaient pour mission de développer une partie des nouveaux produits et des nouveaux process de fabrication, une fois les spécificités générales des produits et des process figées dans leurs grands principes par les équipes de l'ingénierie centrale. Cette intention stratégique, si elle ne résume pas l'ensemble des réflexions ayant conduit à sa formulation, fait tout de même écho à la thèse défendue par Gomez (2013) sur l'invisibilité du travail. Selon cet auteur, la transformation des organisations (depuis les années 80) n'a pu s'accomplir sans la financiarisation du travail lui-même. Financiariser le travail (...) ne signifie pas qu'on repère la contribution de celui-ci au profit, mais qu'on assimile le travail à sa contribution au profit (...). Le travail réel est absorbé par sa contribution au profit. Il doit s'ajuster à un résultat défini a priori et qui dessine le cadre pour l'activité de l'employé. Manager ce travail c'est fixer des objectifs et le maintenir dans les limites ainsi tracées qui assurent sa contribution au profit. Dans notre cas, le raisonnement stratégique de l'entreprise a été initié en considérant que le travail devait s'ajuster à la stratégie de conquête. Nous verrons dans quelle mesure le recours à la simulation organisationnelle dans le cadre de la recherche intervention permettra de lui redonner une plus juste place.

La stratégie de déploiement postulait que la nouvelle organisation devait se déployer en 4 ans et débuter par l'embauche des concepteurs " produit ", les premiers à intervenir en masse durant l'activité de développement puis par les concepteurs "process». Il s'agissait ainsi de créer, par reproduction, une activité équivalente à celle d'une partie de l'ingénierie mère, dans les centres d'ingénierie nouvellement créés à l'international. L'envoi d'expatriés était limité tant en raison de leur coût que de la volonté d'offrir aux ressources humaines locales des places valorisées dans la hiérarchie de la nouvelle organisation. Des référents techniques, au niveau de l'ingénierie mère, étaient repérés et devaient soutenir les centres en création. Le déploiement s'est déroulé dans un premier temps selon le plan proposé mais quelques mois après sa mise en œuvre des surprises et des déconvenues sont apparues sur une des nouvelles entités d'ingénierie.

L'activité des concepteurs de ce centre d'ingénierie à l'international posait problème. Les difficultés les plus importantes étaient la faible qualité des études réalisées par les ingénieurs locaux (ces études devaient être reprises en profondeur par l'ingénierie centrale) et le turnover élevé des équipes nouvellement constituées (un turnover oscillant entre 15 et $25 \%$, et pouvant même atteindre $65 \%$ pour une des équipes). S'il ne faisait aucun doute que la faible qualité des études constituait un problème sérieux pour l'atteinte des objectifs initialement envisagés, le phénomène du turnover était inquiétant, mais difficilement qualifiable. Etait-ce une dynamique «naturelle» dans la phase de constitution d'un centre de développement? Etait-ce dû à une volatilité des ressources, inhérente à la dynamique de croissance économique du pays hôte ? Cela constituait-il un véritable problème ne pouvant se résoudre par un accroissement des embauches pour compenser les départs? L'interprétation restait ouverte. Quoi qu'il en soit, il devenait nécessaire d'analyser finement les causes des dysfonctionnements constatés pour tenter, si ceux-ci s'avéraient problématiques, d'y répondre. 


\subsection{Le dispositif de pilotage et de suivi de la transformation organisationnelle}

Pour structurer la collaboration avec le partenaire industriel nous avons distingué différentes étapes dans le processus de recherche intervention, en reprenant la logique de la démarche CDO présentée plus haut. Nous venons de présenter l'intention stratégique qui dans notre cas constitue l'étape d'explicitation des finalités. Suivront ensuite l'étape d'analyse du fonctionnement organisationnel existant c'est-à-dire de la dynamique organisationnelle initiale, l'étape de conception d'un nouveau schéma formel de l'organisation et d'analyse par anticipation du fonctionnement prévisible de ce nouveau schéma (faisabilité de la nouvelle dynamique organisationnelle), et l'étape de mise en œuvre, de suivi et d'analyse du nouveau fonctionnement réel effectivement obtenu.

Un dispositif de pilotage du projet de transformation organisationnel permettait d'organiser la concertation et la participation tout au long du projet afin de contribuer aux étapes de conceptions et de tirer les enseignements des différentes expérimentations. Il était constitué de plusieurs instances ad hoc ou permanentes (comité de pilotage de la recherche, groupe d'analyse ad hoc, présentation dans des comités de direction et à des équipes transverses, réunions de service métier et journée d'échange par métier) que nous nourrissions d'entretiens menés à différents niveaux de l'organisation. Ces instances permettaient selon les cas de réaliser, de partager et de discuter les analyses et propositions avec différents acteurs à des niveaux variés de l'organisation. L'intensité des interactions avec le terrain s'explique notamment par la présence continue et sur plusieurs années, au sein de l'entreprise partenaire, d'un des chercheurs de l'équipe. Nous reviendrons dans la partie discussion sur la nature plus ou moins participative des interactions et sur la place de la simulation dans celles-ci. Mais revenons à ce stade à l'analyse des difficultés dans le centre d'ingénierie nouvellement créé.

\subsection{L'analyse du travail réel des concepteurs locaux}

Les explications spontanées des difficultés constatées au sein de l'ingénierie locale (niveau de qualification insuffisant à l'embauche, départ pour cause salariale dans un marché de l'emploi en plein essor, caractéristiques culturelles du pays d'accueil de la nouvelle entité), étaient discutables. En effet, les embauches portaient sur des ingénieurs dont les niveaux de formation étaient élevés, ceux qui quittaient l'entreprise ne progressaient pas forcément sur le plan salarial et les différences culturelles ne s'étaient pas révélées gênantes pour toutes les activités d'ingénierie implantées dans ce pays.

Pour comprendre la dynamique organisationnelle existant dans l'ingénierie locale nous avons utilisé la grille d'analyse de la Dynamique Identitaire Globale de L'acteur. La cinquantaine d'entretiens menés sur place, analysée en collaboration avec un expert du métier réalisant un " passage cadre ", mit en évidence que les concepteurs locaux étaient en fait dans l'incapacité de réaliser l'activité déléguée par l'ingénierie mère. Les quelques personnes détenant un début d'expertise en local étaient mobilisées sur la réalisation de tâches trop complexes pour leur niveau de compétence. Elles étaient censées dans le même temps former les nouveaux entrants alors qu'elles étaient elles-mêmes en situation d'incompétence. L'encadrement technique à distance se révélait défaillant, l'expert de 
l'ingénierie mère, trop pris par d'autres activités, n'assurait pas son rôle de soutien. La hiérarchie locale ne se sentait pas légitime pour refuser les nouvelles attributions de travaux à réaliser, d'autant qu'elle n'était pas toujours en mesure d'évaluer l'adéquation entre le niveau de complexité de ceux-ci et le niveau de compétence de ses équipes. Enfin l'évaluation négative de la qualité du travail local (régulièrement renvoyée par l'ingénierie centrale) sapait progressivement la confiance en eux des concepteurs locaux. Ces derniers se sentaient au fil du temps incompétents et ce sentiment les conduisait soit à quitter leur entité dans le cadre d'une mobilité interne, soit à définitivement quitter l'entreprise, ou encore à rester tout en se désinvestissant.

Cette analyse conduisit à considérer que la cause majeure du turnover observé résidait dans la mise en incompétence des concepteurs locaux. L'origine du problème était la faiblesse de l'encadrement technique local (car le marché du travail n'offrait pas de ressources expérimentées sur les métiers concernés) plutôt qu'une incapacité intrinsèque des jeunes ingénieurs à apprendre le métier. Mais si l'étiologie globale du problème observé paraissait identifiée, il restait de nombreuses questions à traiter. Combien d'expatriés fallait-il envoyer? Combien fallait-il embaucher de nouvelles recrues et selon quelles temporalités? Pouvait-on prévoir la temporalité du processus global de croissance et de montée en compétence? Au bout de quelle période le centre de développement serait capable de devenir autonome pour effectuer le travail de développement que les stratèges avaient prévu de lui attribuer?

\subsection{Révéler les processus d’apprentissage du métier}

Si le problème provenait du blocage des dynamiques d'apprentissage des concepteurs locaux, il renvoyait alors à une question essentielle, finalement très simple, mais sans réponse dans l'entreprise : comment un concepteur apprend-il son métier? L'analyse de l'ingénierie locale était intéressante pour mettre en évidence les difficultés mais devait être comparée à une situation de référence où l'apprentissage était possible. Cela nous a conduit à réaliser une analyse comparative des dynamiques d'apprentissage de l'ingénierie mère et de l'ingénierie locale.

Nous présentons ici une synthèse de cette analyse. Tous les éléments qui suivent ont été explicités en collaboration avec un concepteur expérimenté sur la base d'entretiens avec certains membres du métier. Ils ont été validés, dans leurs principes généraux, lors de plusieurs séminaires avec l'ensemble des représentants du métier concerné. Ils ont également été présentés et discutés avec le chef de service métier. En définitive, assumer un rôle de concepteur nécessite la maîtrise de savoirs complexes: des fondamentaux théoriques comme par exemple le calcul en éléments finis, la maitrise du fonctionnement des outils numériques, la capacité à sélectionner des méthodes pour analyser une prestation donnée, la capacité à analyser les résultats de ces méthodes, le fait de tenir compte de l'impact d'une prestation sur l'ensemble des prestations et des contraintes des autres métiers, inventer de nouvelles méthodes, etc. L'acquisition des compétences techniques est également fortement dépendante du niveau et de la nature des compétences du collectif entourant l'individu. Ainsi, il est possible de styliser les différentes étapes de la montée en compétence d'un jeune concepteur en distinguant cinq rôles, en fonction à la fois des compétences à acquérir et de la nature du collectif environnant permettant l'apprentissage à chaque niveau. Ces cinq rôles représentent les étapes du processus d'apprentissage. 
Tableau 1 : Parcours d'apprentissage et nature du collectif nécessaire à la montée en compétence. Chart 1: Learning pathway and nature of the collective needed for skill improvement

\begin{tabular}{|c|l|l|}
\hline Rôle & \multicolumn{1}{|c|}{ Compétence } & \multicolumn{1}{|c|}{$\begin{array}{c}\text { Nature du collectif } \\
\text { permettant l'apprentissage }\end{array}$} \\
\hline Apprenti & $\begin{array}{l}\text { Fondamentaux théoriques et } \\
\text { outils numériques }\end{array}$ & Encadrement par un sénior \\
\hline Junior & $\begin{array}{l}\text { Faire tourner un modèle } \\
\text { numérique sur une prestation }\end{array}$ & Encadrement par un sénior \\
\hline Senior & $\begin{array}{l}\text { Analyser une prestation en tenant } \\
\text { compte des autres prestations }\end{array}$ & $\begin{array}{l}\text { Encadrement par un expert projet et } \\
\text { le collectif de séniors compétents sur } \\
\text { les autres prestations du même } \\
\text { métier }\end{array}$ \\
\hline $\begin{array}{l}\text { Expert } \\
\text { Projet }\end{array}$ & $\begin{array}{l}\text { Prendre en compte toutes les } \\
\text { prestations de son métier et gérer } \\
\text { les contraintes des autres métiers }\end{array}$ & $\begin{array}{l}\text { Soutien par un expert métier, le } \\
\text { collectif d'experts projet sur son } \\
\text { métier, et le collectif d'experts des } \\
\text { autres métiers }\end{array}$ \\
\hline $\begin{array}{l}\text { Expert } \\
\text { Métier }\end{array}$ & Inventer de nouvelles méthodes & Echange entre pairs \\
\hline
\end{tabular}

31 Cette analyse permet de répondre à un certain nombre de questions initialement posées et en soulève d'autres. Elle confirme le besoin d'encadrement technique devant être assuré par des expatriés sur les activités de conception complexes lorsque le marché local n'offre pas de ressources expérimentées. Elle pose la question du nombre d'expatriés sachant qu'il dépend du nombre de nouveaux embauchés que l'on souhaite recruter et pointe le lien existant entre le rythme des embauches, la vitesse de formation des nouveaux entrants et le temps nécessaire à ces derniers pour eux-mêmes devenir des encadrants techniques. Étant donné la temporalité des apprentissages individuels (environ une dizaine d'années ${ }^{3}$ pour passer du rôle de junior au rôle d'expert métier), l'analyse réalisée met en évidence que le processus de création d'une entité de développement à l'international s'inscrit dans un temps bien plus long que ce qui avait été initialement envisagé.

Ces éléments sont déjà très utiles mais ils sont trop compliqués pour aisément projeter et calculer la dynamique de création d'un centre d'ingénierie. De la même façon une expérimentation "in-vivo» serait déraisonnable au regard de la temporalité du processus et de ces enjeux. Pour autant il serait d'un grand secours de pouvoir répondre aux questions suivantes, même de façon approchée: À quel rythme effectuer les embauches? Comment évaluer le délai de montée en «compétence collective " pour la nouvelle entité au regard du délai de montée en compétence des individus? Quel niveau de complexité de l'activité l'ingénierie centrale doit-elle déléguer à l'ingénierie locale et selon quelle temporalité ? Quel est l'impact du turnover sur cette dynamique ? Et c'est à ce stade de notre raisonnement que le recours à la simulation devient nécessaire.

\subsection{Modélisation et paramétrage d'une dynamique organisationnelle}

Pour passer à la simulation nous avons construit, sur la base de l'analyse qualitative de l'ensemble des métiers ${ }^{4}$, un modèle simplifié de la dynamique d'apprentissage collective. 
Chaque individu, sur chacun des métiers, progresse sur une échelle de cinq niveaux de compétence en fonction de la complexité des activités de conception. La progression d'un individu, d'un niveau au suivant, est conditionnée par la présence des expertises au sein du collectif qui l'entoure. Par exemple un expert projet ne progressera qu'en présence d'un expert métier au sein de son propre métier et d'experts projet sur les métiers connexes. Les capacités et les besoins d'encadrement pour chaque niveau de compétence au sein de chaque métier et les conditions de coprésence entre métiers ont été paramétrés $\left(a, b, b^{\prime}, b^{\prime \prime}, \ldots\right)$ sur la base des échanges menés avec les concepteurs de chacun des métiers. En définitive, la modélisation de l'architecture des liens d'apprentissage peut se représenter comme suit :

Figure 1 : Architecture des liens d'apprentissage.

Figure 1: Architecture of the learning links

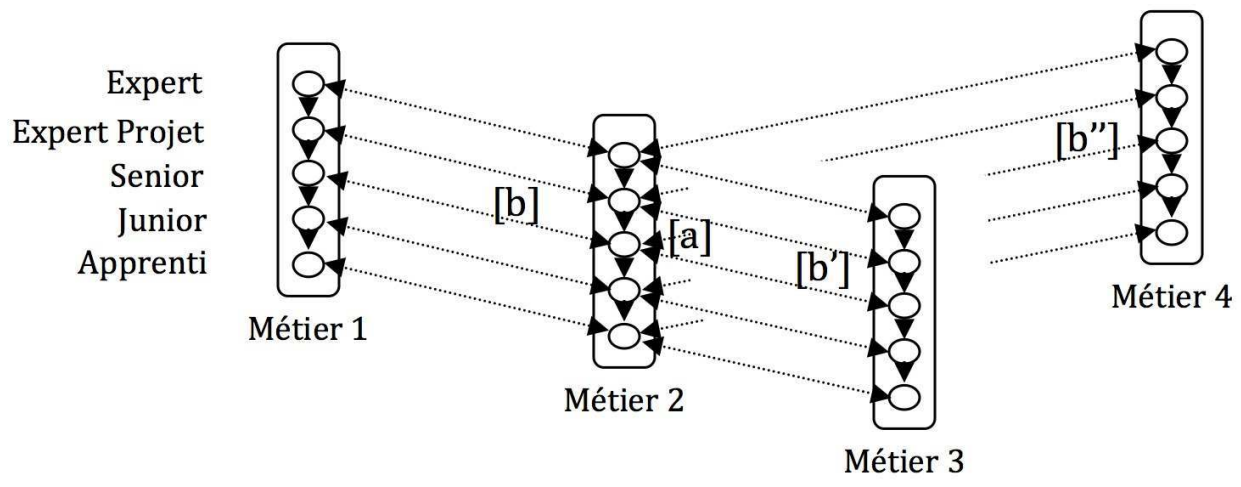

Le temps moyen de progression d'un individu, lorsque les conditions d'intégration et d'apprentissage sont assurées, a été estimé sur la base de la progression en compétence des concepteurs de l'ingénierie mère. Il se synthétise sous la forme d'une courbe de progression croissante et concave (cf. Figure 2). Ce qui signifie que les durées d'apprentissage sont plus longues à mesure que l'on progresse vers des niveaux de compétence élevés.

Figure 2 : Temporalité des apprentissages individuels.

Figure 2: Time needed for an individual to progress

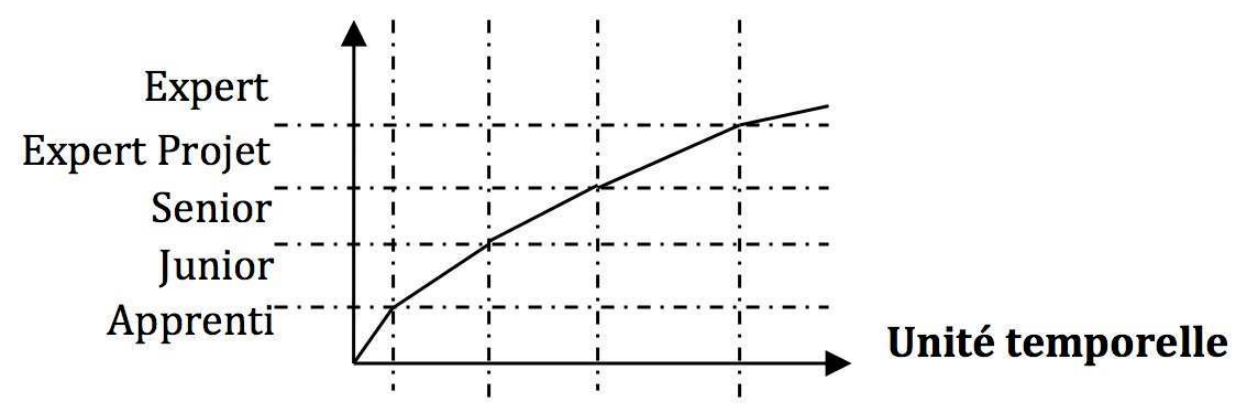

Le collectif de concepteurs prendra en charge, selon sa robustesse technique, des activités de conception de plus en plus complexe (D, C, B, A). Lorsqu'il n'y a que des apprentis et des juniors, l'entité d'ingénierie ne peut réaliser que des activités support (niveau D). La présence d'au moins un senior dans chaque spécialité permet d'assumer des activités 
d'adaptation légère de produits déjà existants (niveau C). Un expert projet dans chacune des spécialités métiers offre la possibilité de réaliser le développement d'un produit dont $65 \%$ du design a été spécifié (niveau B). Enfin, lorsqu'un expert métier est présent dans chaque spécialité, l'entité est en mesure de développer un produit complet (le niveau A).

\subsection{Simulation d'une dynamique organisationnelle}

Cette modélisation, relativement simple dans ses principes, permet de simuler la dynamique de création et de croissance d'une entité d'ingénierie. En faisant tourner le modèle en ayant fixé le nombre d'expatriés nous pouvons estimer la quantité maximum de nouveaux embauchés qu'il est possible d'intégrer dans le temps, la capacité d'encadrement des concepteurs locaux en fonction de leur apprentissage effectif et en définitive le temps nécessaire pour que l'entité d'ingénierie soit en mesure de réaliser les quatre grands types d'activités.

Dans l'exemple ci-dessous, 75 unités de temps permettent de construire une équipe de 600 concepteurs pouvant collectivement réaliser l'activité la plus complexe. Les pics bleus sur le graphique nous indiquent le nombre maximum et la temporalité des embauches au regard de l'utilisation maximum des capacités d'encadrement. Cette simulation donne ainsi la croissance potentielle maximum dans le temps en fonction des capacités d'expatriation de l'ingénierie centrale (Figure 3).

Figure 3 : Simulation du processus de création sans turnover. Figure 3: Simulation of the creation process without staff turnover

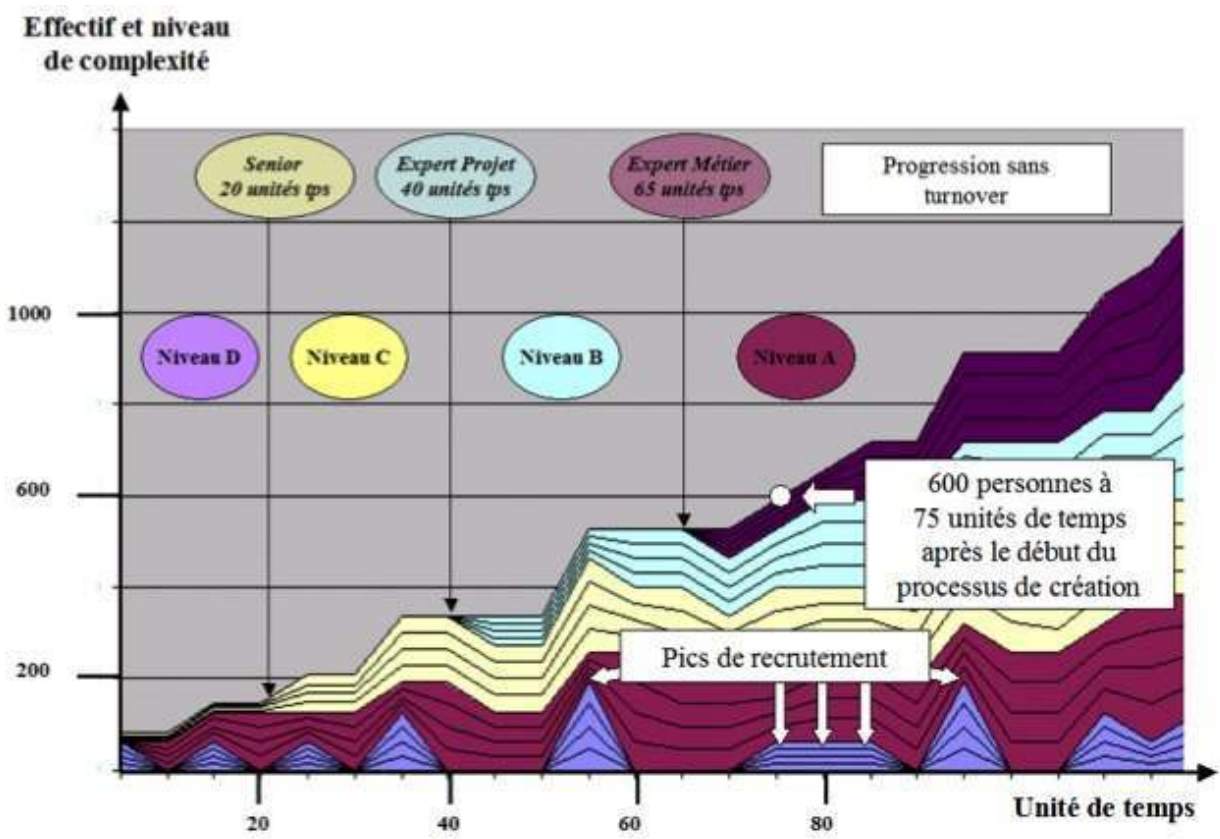

L'un des intérêts de cette simulation est d'appréhender, à partir de ces premiers résultats, l'effet du turnover sur la dynamique de croissance du collectif de concepteur. Nous avons introduit un risque de départ au moyen d'une variable aléatoire indépendante pour chaque individu. Si malgré la perte de ressources liée à ces départs il reste des capacités d'encadrement excédentaires, des embauches de juniors viennent la compenser jusqu'à saturation des encadrants. Si la perte de ressources conduit à des capacités 
d'encadrement sursaturées, des départs de ressources les moins expérimentées viennent rétablir l'équilibre. Cela nous a permis d'estimer l'impact du turnover sur la progression d'une entité d'ingénierie dans ce contexte international. Le schéma ci-dessous représente la déformation du processus précédent en introduisant $10 \%$ de turnover. Nous voyons, sur la Figure 4, que 75 unités de temps ne permettent plus d'atteindre que 500 personnes par rapport aux 600 personnes du processus sans turnover et que l'atteinte du niveau qualitatif le plus élevé (niveau A) nécessite 15 unités de temps supplémentaires.

Figure 4 : Simulation du processus de création avec $10 \%$ de turnover. Figure 4: Simulation of the creation process with a staff turnover of $10 \%$

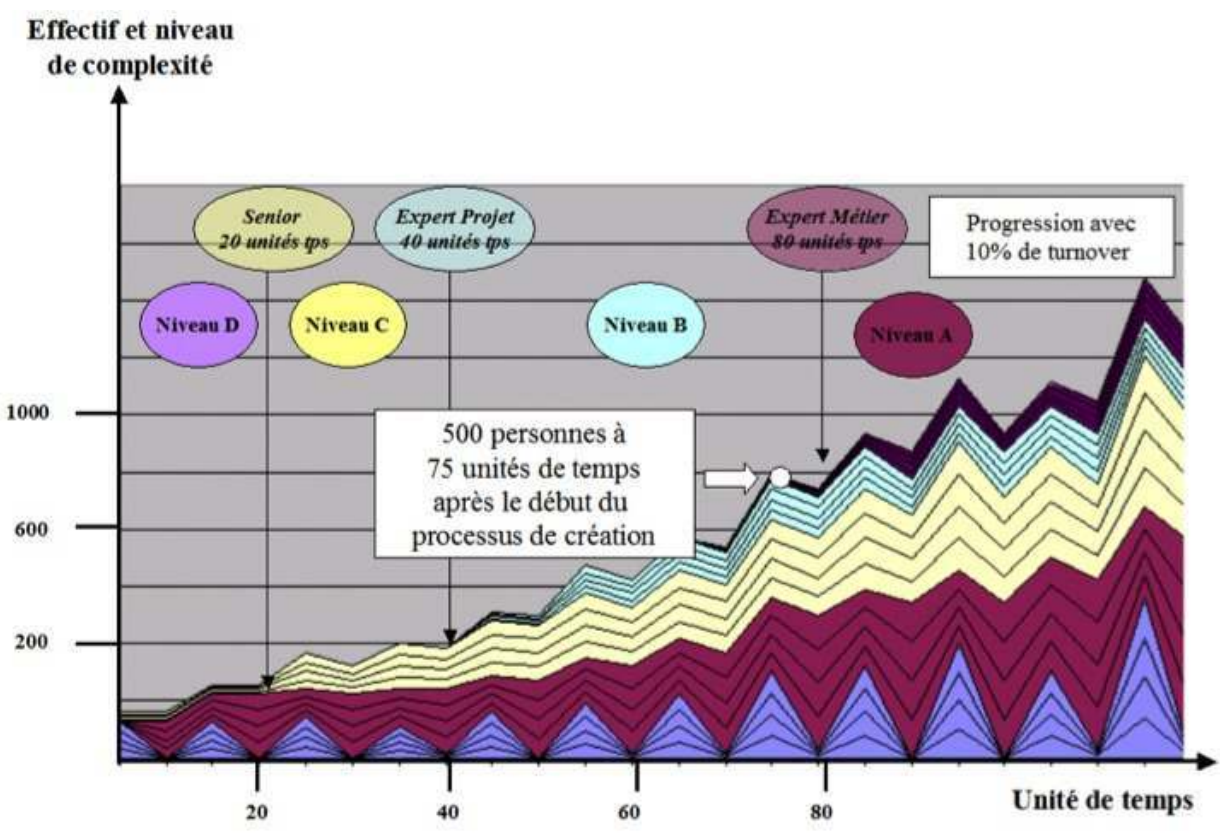

Le double effet du turnover (l'effet direct qui chaque année réduit de $10 \%$ la taille du collectif et l'effet indirect qui du fait des départs aléatoires de concepteurs expérimentés conduit au départ par manque d'encadrement des concepteurs moins expérimentés) n'est pas une surprise pour le modélisateur quant à son principe mais renseigne sur son ampleur. La surprise (sur le principe et sur l'ampleur) est potentiellement plus importante pour les membres de l'organisation, notamment le fait qu'il est impossible de compenser les départs par des embauches supplémentaires. Plus le turnover affectera les ressources ayant un niveau de compétence élevé, plus l'effet de propagation sera important puisque ce sont elles qui détiennent la capacité de formation des jeunes entrants. La simulation révèle ainsi les effets variés du turnover et surtout leur ampleur selon la maturité du centre de développement. Si au début du processus il est pertinent de lutter contre le turnover par un accroissement des embauches, cette stratégie devient contre-productive après une première étape de maturité.

Concernant la complexité de la conception réalisable dans l'entité d'ingénierie à l'international, la simulation permet d'appréhender les effets du turnover sur le temps nécessaire pour atteindre les différents niveaux de complexité de l'activité (D, C, B, A). Si le niveau de turnover a relativement peu d'impact sur l'atteinte d'une capacité de conception de niveau D, il n'en est pas de même pour le niveau de complexité supérieur. Si le seuil de $10 \%$ de turnover est dépassé, l'impact sur le temps nécessaire pour réaliser une conception complexe augmente très sensiblement ( $47 \%)$. Il faut donc s'efforcer de 
rester nettement en dessous de ce seuil. Si on ajoute le fait, comme nous l'avons mis en évidence précédemment, que $10 \%$ de turnover réduit la taille du collectif de conception, on en arrive à préconiser de viser à maintenir le turnover en dessous d'un seuil de $5 \%$.

\subsection{Les apports de la simulation en design organisationnel pour quel mode de participation?}

41 L'élément peut être le plus marquant concernant les effets de la simulation de la dynamique de création d'un centre de développement à l'international concerne les décisions qui ont suivi. Suite à la présentation de ces travaux, le sommet stratégique a autorisé qu'un nombre conséquent d'expatriés renforce l'ingénierie locale et le nombre d'embauches initialement prévues a été fortement revu à la baisse (passant sur la période de temps et le périmètre concernés de 300 à 150 personnes). La complexité des activités déléguées a également été revue à la baisse. En outre les embauches n'étaient plus réalisées de manière séquentielle (concepteurs produit en premier, puis concepteurs process) mais en même temps. Dans les périodes qui ont suivi, ce nouveau dimensionnement du processus de création a conduit à une diminution très importante du turnover, passant de 15 à $20 \%$ en moyenne à moins de $3 \%$. Cette nouvelle phase d'expérimentation in vivo a confirmé à la fois l'hypothèse d'un turnover ayant comme cause principale le blocage des dynamiques d'apprentissage mais aussi la pertinence des conditions permettant de rendre les apprentissages possibles et la longue temporalité dans laquelle s'inscrit la création d'un centre d'ingénierie.

Mais l'apport de la simulation ne s'est pas résumé à cette inflexion de la stratégie. Elle a également, du fait de la participation des concepteurs au processus d'analyse et de reconception de l'organisation, enrichi la connaissance de ceux-ci sur leur propre métier. Les ingénieurs du centre à l'international, de par les entretiens menés, les restitutions réalisées, le travail d'analyse partagé lors des séminaires et des journées d'échange regroupant l'ensemble des membres du métier se sont progressivement construit une vision très différente des difficultés qu'ils avaient vécues. De la même manière, les débats sur les mécanismes d'apprentissage, les discussions, sur les différents chemins possibles conduisant un individu d'un statut de jeune entrant à une compétence d'expert métier, ont accru la vitalité du métier. La modélisation, du fait de la simplification qu'elle appelle, ne rend pas visible la modification du regard de chaque individu sur son propre métier, ni le renforcement du collectif métier que le processus global a permis. Pour les acteurs métiers, ce n'est donc pas la simulation qui est un apport mais le chemin permettant de constituer une base empirique robuste. Cette base empirique constituée, discutée, partagée ne conduit plus vraiment à des surprises pour les membres du métier si ce n'est la surprise de la traduction d'un univers métier décrit dans le langage du métier, en un univers métier décrit dans le langage des stratèges.

Concernant les stratèges, il est nécessaire de distinguer deux cas. Ceux étant partie prenante du processus de recherche-intervention (ne serait-ce que parce qu'ils nous ont demandé d'intervenir dans leur département) et « le groupe stratégique », composé d'un représentant de chaque département, en charge du déploiement de l'ingénierie à l'international.

Les stratèges partie prenante étaient impliqués dans le processus. S'ils n'ont pas participé pour des raisons pratiques à l'ensemble des explorations, les synthèses des analyses qualitatives, les différentes étapes de construction et les résultats de la simulation leurs 
ont été présentés. La synthèse finale n'a pas été une surprise du fait de la progressivité de la constitution des connaissances. Ce sont d'ailleurs eux qui sont allés défendre l'inflexion stratégique au sommet de l'entreprise.

Pour le « groupe stratégique » il n'y a pas eu de participation. Les analyses et les résultats ont été présentés mais l'accueil de ceux-ci laissait une grande place au doute. Si l'étude portait sur le plus gros des départements en termes d'effectif ( $10 \%$ de l'ingénierie) la validité des analyses pour les autres départements pouvait être légitimement questionnée. En outre, le département d'accueil de la recherche intervention avait anticipé la stratégie d'internationalisation et était en avance par rapport aux autres qui n'avaient pas encore éprouvée concrètement les premières difficultés. Cet accueil réservé est resté sans suite mais une analyse comparée des différents départements menée quelques années plus tard semble indiquer que la généralisation était belle et bien possible.

Enfin, concernant les acteurs du déploiement, un travail très fin de conception du processus de croissance a été mené. De nombreuses réunions de travail ont été nécessaires pour tirer tous les enseignements des analyses sur la montée en compétences. Pour certains acteurs, malgré l'accord de la hiérarchie, réduire les embauches et accroitre le nombre d'expatriés a été très difficile à accepter. Cette proposition était tellement différente du scénario initialement communiqué par l'entreprise qu'il a fallu de longues explications pour partager le bien fondé du nouveau processus. D'autres acteurs au contraire étaient convaincus bien avant que nous débutions le travail sur le déploiement de détail.

\section{Conclusion}

En définitive, la manière dont nous avons mobilisé la simulation est cohérente avec notre démarche conception et dynamique des organisations. Dans nos pratiques intervenantes nous adoptons une approche concourante avec d'importantes rétroactions entre la conception formelle de l'organisation et l'analyse de la dynamique organisationnelle. Nous mettons en œuvre une démarche participative tout en assumant de disposer de savoirs méthodologiques, d'analyse et de conception formelle certes partiels mais utiles aux membres de l'organisation. Il en est de même dans notre usage de la simulation faiblement prédictive. Il apparait d'ailleurs que c'est la participation dans le processus de constitution d'une base empirique étoffée permettant la simulation qui a été l'élément permettant aux acteurs de s'approprier la pertinence de la simulation pour leur activité. Nous opérons ainsi à la fois comme coanalyste de l'activité, coconcepteur de l'organisation formelle et comme traducteur du langage métier en langage stratégique pour rendre le travail à nouveau visible. 


\section{BIBLIOGRAPHIE}

Daniellou, F. (2007). Des fonctions de la simulation des situations de travail en ergonomie, Activités, 4-2.

Daniellou, F., \& Béguin P. (2004). Méthodologie de l'action ergonomique : approches du travail réel », Ergonomie. Presses Universitaires de France, pp. 333-358.

Gomez, P-Y. (2013), Le travail invisible, enquête sur une disparition, François Bourin. Éditeur.

Hubault, F., \& Bourgeois, F. (2004). Disputes sur l'ergonomie de la tâche et de l'activité, ou la finalité de l'ergonomie en question, Activités, 1-1.

Lerch, C. (1998). Une Nouvelle Représentation du Contrôle Organisationnel : le Pilotage des Processus, Thèse de Doctorat de Sciences de Gestion. Université Louis Pasteur, Strasbourg.

Lorino, P. (2009). Concevoir l'activité collective conjointe : l'enquête dialogique. Étude de cas sur la sécurité dans l'industrie du bâtiment, Activités, 6-1.

Peyrolle, J-C., \& Lorino, P. (1999). Enquête sur le facteur X. L'autonomie de l'activité pour le management des ressources humaines et pour le contrôle de gestion. Revue de Gestion des Ressources humaines, no 33

Reynaud, J-D. (1997). Les Règles du jeu : L'action collective et la régulation sociale, Armand Colin, Paris.

Salembier, P., \& Pavard, B. (2004). Analyse et modélisation des activités coopératives situées. Évolutions d'un questionnement et apports à la conception, Activités, 1-1.

Sardas, J-C., Dalmasso, C., \& Lefebvre, P. (2011). Les enjeux psychosociaux de la santé au travail, des modèles d'analyse à l'action sur l'organisation, Revue Française de Gestion, vol. 37/214 pp. 69-88.

De Terssac, G. (2002). Le travail : une aventure collective, Toulouse. Octarès.

Schwartz, Y. (2007). Un bref aperçu de l'histoire culturelle du concept d'activité. Activités, 4 (2), pp. 122-133.

\section{NOTES}

1. Notons que l'usage du terme cognitif nous semble restrictif pour parler du processus de construction des connaissances. En effet, si la part du cognitif dans ce processus est indéniable, l'investissement subjectif l'est tout autant.

2. La construction d'un modèle est la formalisation (c'est à dire la définition des variables d'entrée et de leurs interactions, et définition des critères de résultat) du phénomène à étudier. Cette modélisation s'effectue d'abord de façon purement conceptuelle sur le papier (modèle conceptuel) puis sur un support (physique ou informatisé) qui va permettre la simulation, c'est pourquoi on parle alors de modèle de simulation). La simulation va ensuite consister à «faire tourner le modèle » pour tester différentes configurations de variables d'entrée, pour évaluer et comparer les résultats obtenus. 
3. Pour des raisons de confidentialité nous ne donnons pas ici le détail des temps d'apprentissage pour chaque rôle, mais il nous a bien entendu fallu les estimer.

4. L'analyse des autres métiers n'est pas présentée dans ce papier pour ne pas trop développer le propos. On voit néanmoins qu'il a fallu modéliser et paramétrer les conditions de co-présence entre niveaux équivalents de chaque métier.

\section{RÉSUMÉS}

La simulation en sciences sociales s'ancre dans des traditions de recherche dont le genre disciplinaire et le style des chercheurs colorent l'usage. Technologie à appliquer pour les uns, techniques permettant de favoriser les débats pour d'autres, les possibilités offertes sont protéiformes. Dans ce papier nous présentons une façon originale de mobiliser la simulation, en situation de recherche-intervention, dans le domaine des sciences de gestion. Après avoir discuté de la différence que nous voyons entre enquête dialogique et démarche conception et dynamique des organisations, nous présentons une manière d'utiliser une simulation organisationnelle afin d'articuler le raisonnement stratégique d'une entreprise, la conception et le déploiement d'une nouvelle organisation et l'activité des acteurs de terrain. Ce faisant, nous explorons la possibilité d'opérer une meilleure intégration entre les décisions stratégiques prises par les managers de haut niveau, les choix opérés par le management intermédiaire quant à la stratégie de déploiement et la manière dont l'activité est effectuée et vécue par les travailleurs (intellectuels dans le cas que nous allons traiter) qui en ont la charge. Nous discutons donc, à l'heure où l'on s'interroge sur l'invisibilité du travail (Gomez, 2013), les moyens susceptibles de lui redonner une place dans le processus de décision stratégique et de design organisationnel des grandes entreprises.

Simulation in social sciences entrenches itself in research traditions whose disciplinary type and researcher style color its purpose. For some, the technology to be applied, for others the techniques that encourage debate - the possibilities are multifaceted. In this paper, we present an original way of mobilizing simulation, in a situation of collaborative research in the field of management sciences. After discussing the difference between dialogical investigation and the organizational design and dynamic of organizations approach, we show how organisational simulation can be used to articulate a company's strategic reasoning, a new organizational design and deployment, and the activity of actors in the field. In so doing, we explore the possibility of operating improved integration between strategic decisions taken by high-level managers, the choices made by middle management concerning the deployment strategy, and how the activity is performed and experienced by the workers (intellectual in this case) who are responsible for it. At a time when we are reflecting upon its invisibility (Gomez, 2013), we thus discuss possible ways of bringing work back into the strategic decision-making and organizational design process in large companies. 
INDEX

Keywords : organizational design, organizational dynamics, simulation, engineering, internationalization

Mots-clés : design organisationnel, dynamique organisationnelle, simulation, ingénierie, internationalisation

\section{AUTEURS}

\section{CÉDRIC DALMASSO}

Mines ParisTech, PSL Research University, CGS - I3 - UMR CNRS 9217

60 boulevard Saint Michel, 75272 cedex 06

cedric.dalmasso@mines-paristech.fr

JEAN-CLAUDE SARDAS

Mines ParisTech, PSL Research University, CGS - I3 - UMR CNRS 9217

60 boulevard Saint Michel, 75272 cedex 06

jean-claude.sardas@mines-paristech.fr 